\title{
FLOW-CHAMBER MICROARRAY DETECTION OF MALARIAL ANTIGENS
}

\author{
Daniel J. Laser, Corwin T. Hardham, and Johann Kim \\ Wave 80 Biosciences, Inc. \\ $23253^{\text {rd }}$ St, Suite 215, San Francisco, California 94107
}

\begin{abstract}
Detecting and quantifying circulating proteins produced by malarial parasites provides valuable information to guide diagnosis and treatment. We report on the detection and quantification of the malarial antigens HRP-II and aldolase with single-use microfluidic cartridges incorporating protein microarrays. The microarray-based cartridges are suitable for use with handheld bioassay instruments. Reagent and sample transport in the cartridges is controlled by integrated silicon electroosmotic micropumps. Applications of the flow-chamber microarrays for experimentally determining binding parameters for pairs of biological molecules are explored.
\end{abstract}

\section{INTRODUCTION}

Historically, body fluid analysis for malaria diagnosis has been through microscopic examination of blood smears for parasites, through automated analysis with laboratory PCR systems; or by card-type kits [1]. While these methods are satisfactory for many clinical settings, in some instances a fully automated (e.g. no manual dispensing/metering of reagents or skilled interpretation of assay outcomes), highly portable system for detecting and quantifying malarial antigens is desirable.

We sought to leverage protein microarrays to develop new tools and techniques for detecting and quantifying malarial antigens. Microarrays (both DNA and protein) are well established for drug discovery, but are underutilized in portable medical diagnostics. For such applications, it is convenient to locate microarrays within microscale reaction chambers linked to channel networks for delivering samples and reagents. Abundant options now exist for controlled sample/reagent delivery [2]. However, selection of geometries and operating parameters for flow-chamber microarrays requires careful study of the interplay between biochemical and microfluidic effects. The methods introduced here for developing microarray malarial antigen assays are applicable to a wide range of analytes.


Figure 1: Left: Photograph of reaction chamber with protein microarray. The microarray (typically eight spots) is printed on a nitrocellulose film. Right: photomicrograph of a portion of a reacted microarray.

\section{EXPERIMENTAL METHODS}

Flow-chamber microarrays of the design shown in figure 1 were used in experiments. The microarray-containing reaction chambers are built into microfluidic cartridges; the cartridges, along with a handheld instrument, compose an automated bioassay system, a prototype of which is illustrated in figure 2. To run an assay, a small ( $<50$ microliters) whole blood sample, obtained through e.g. fingerstick, is allowed to wick into the cartridge sample pad (inset of figure). The sample pad is sealed by sliding closed the cartridge cover, after which the cartridge is inserted into the handheld instrument (overall size $310 \mathrm{~cm}^{3}$, weight incl. batteries $292 \mathrm{~g}$ ). Reagent and sample transport within the cartridge is driven by integrated slit capillary array silicon electroosmotic micropumps [3], which in turn are controlled by electrical signals from the handheld.

The reaction chamber cross-sectional dimensions are $120 \mu \mathrm{m}$ x $3 \mathrm{~mm}$. The top surface is injection molded cyclic olefin copolymer (COC); the side walls are laser-cut polymer; and the bottom surface is nitrocellulose-coated glass. Molded COC surfaces provide a smooth transition between the reaction chamber and the inlet/outlet microchannels (1 $\mathrm{mm} \times 1 \mathrm{~mm}$ cross-section). Prior to cartridge assembly, a capture microarray (typically eight spots) is printed onto the nitrocellulose film using a modified MicroCaster handheld microarrayer (Whatman) using the manufacturer's standard arraying solutions.

For assay development and characterization, malarial reagents, including monoclonal $\mathrm{IgG}$ and $\operatorname{IgM}$ anti- $P$. falciparum and recombinant $P$. falciparum HRP-II antigen, were purchased from Immunology Consultants Laboratory, Newberg, OR.

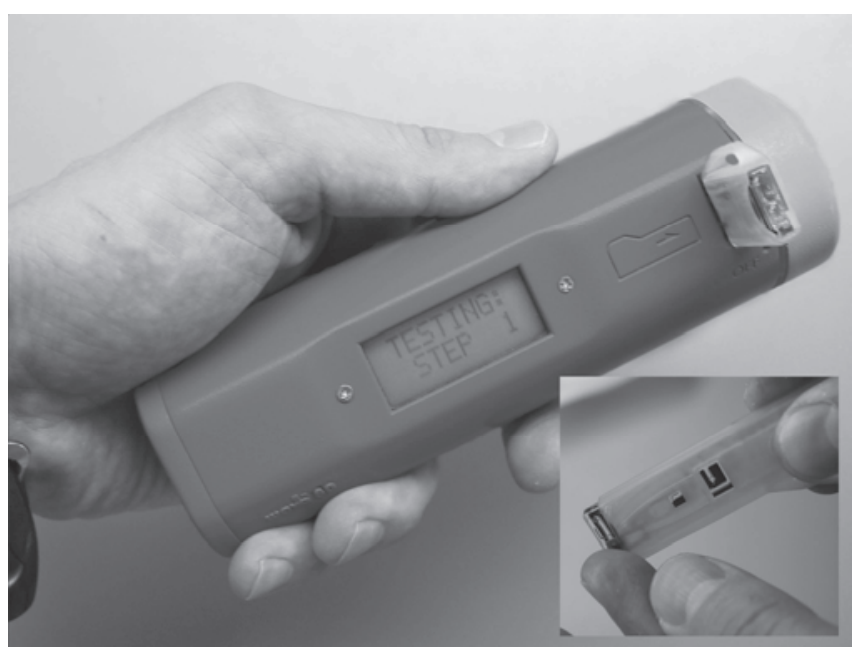

Figure 2: Highly portable, fully automated system for determining the composition of body fluids. 
Malarial antibodies were biotinylated according to standard protocols [4]. Biotinylated goat anti-mouse IgG (Sigma) and Cy2streptavidin (Amersham/GE Healthcare) were used in flowchamber microarray characterization experiments.

\section{ASSAY CHARACTERIZATION AND MICROFLUIDICS MODELING}

Sandwich immunoassays were developed for HRP-II (from $P$. falciparum) and aldolase, a pan-malarial antigen. Data from the development of the HRP-II immunoassay is plotted in figure 3. All combinations of IgG and IgM antibodies were found to yield satisfactory results except for IgM-IgM. Accordingly, HRP-II microarrays include both IgM and IgG spots; IgG is used for labeling. An assay for aldolase was developed in a similar manner. For the aldolase assay development, affinity purified polyclonal antibodies as well as monoclonal antibodies were evaluated.

A model biochemical system of solid-phase biotinylated goat anti-mouse $\operatorname{IgG}$ and fluid-phase Cy2-streptavidin was used to validate a method for characterizing flow-chamber microarray binding as a function of various environmental and fluid mechanical parameters. Figure 4 shows the intensity of anti-IgG spots after flowing through $20 \mu \mathrm{L}$ of Cy2-streptavidin $\left(5 \mu \mathrm{g} \mathrm{mL}^{-1}\right)$ at flow rates of 2,10 , and $50 \mu \mathrm{L} \mathrm{min}{ }^{-1}$ (corresponding to average velocities within the reaction chamber of $0.09,0.46$, and $2.3 \mathrm{~mm}$ $\mathrm{sec}^{-1}$, respectively) at room temperature. In all cases, the flow is fully developed in at least $99 \%$ of the reaction chamber. Analyte flux equations are easily written in terms of the analyte diffusion coefficient and bulk concentration and the one-dimensional velocity profile. The boundary condition at the film-coated surface is defined in terms of the analyte binding (association) rate constant $k_{\mathrm{a}}$. The system is usually solved by numerical methods similar to those described by Myszka et al. [5] and others, with the added model complexity of step-function spatially variations in receptor concentration. Intensity-reading calibration permits calculation of parameters such as $k_{\mathrm{a}}$ from experimental data.

Development of the flow-chamber HRP-II and aldolase assays entailed characterization of binding for antigen capture and labeling steps. Characterization of binding processes for

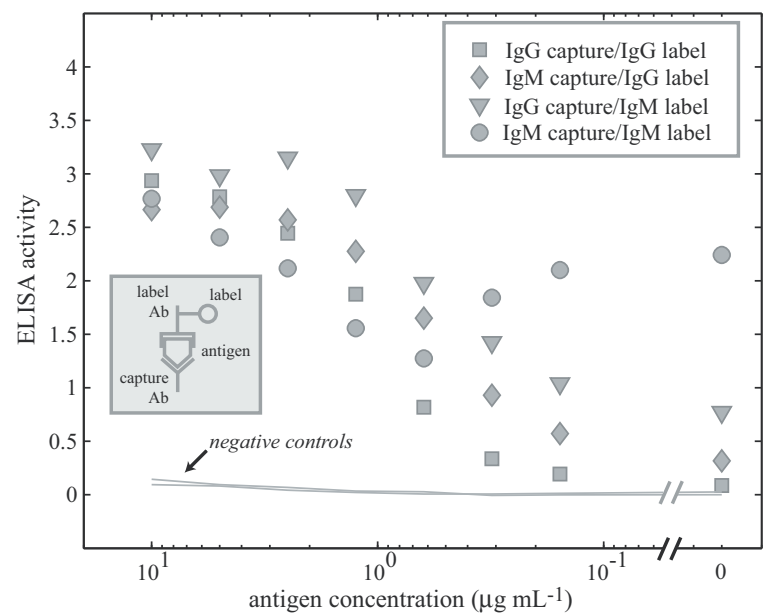

Figure 3: Dose response for the malarial antigen HRP-II (P. falciparum) using mouse $\operatorname{Ig} G$ and $\operatorname{Ig} M$ monoclonal antibodies for capture and labeling. Data shown is for biotinylated label antibody, streptavidin-horseradish peroxidase secondary label. Inset illustrates schematically the principle of sandwich immunoassays.



Figure 4: Microarray capture of Cy2-streptavidin with biotinylated anti-IgG in a flow chamber. Plotted values are the mean intensities of three spots per microarray; error bars show spread. Data shown is for $20 \mu \mathrm{L}$ of Cy2-streptavidin at $5 \mu \mathrm{g} \mathrm{mL} \mathrm{L}^{-1}$; followed by $50 \mu \mathrm{L}$ wash solution; experiments carried out at room temperature.

positive/negative control spots in the microarray permits the use of these spots to calibrate for operational uncertainties.

\section{CONCLUSIONS}

New techniques and tools for detecting and quantifying the malarial antigens HRP-II and aldolase have been developed. Sandwich immunoassays have been developed and adapted for single-use microfluidic cartridges incorporating flow-chamber microarrays. The cartridges are designed to function as part of highly portable bioassay systems. Methods for modeling coupled microfluidic and biochemical processes and experimentally determining reaction rate constants have been developed and refined.

\section{ACKNOWLEDGEMENTS}

This work was supported in part by SBIR contract H9222204-C-0032, U.S. Special Operations Command. It made use of National Nanofabrication Users Network facilities funded by the National Science Foundation under award ECS-9731294.

\section{REFERENCES}

[1] G. A. Farcas, K. J. Y. Zhong, F. E. Lovegrove, C. M. Graham, and K. C. Kain, "Evaluation of the Binax Now ICT Test versus Polymerase Chain Rection and Microscopy for the Detection of Malaria in Returned Travelers," Am. J. Trop. Med. Hyg., vol. 69, pp. 589-592, 2003.

[2] D. J. Laser and J. G. Santiago, "A Review of Micropumps," Journal of Micromechanics and Microengineering, vol. 14, pp. R35-R64, 2004.

[3] D. J. Laser, K. E. Goodson, J. G. Santiago, and T. W. Kenny, "High-Frequency Actuation with Silicon Electroosmotic Micropumps," Proc. 2002 Solid-State Sensors, Actuators, and Microsystems Workshop, Hilton Head, S.C., pp. 231-234.

[4] G. T. Hermanson, Bioconjugation Techniques. San Diego: Academic Press, 1996.

[5] D. G. Myszka, X. He, M. Dembo, T. A. Morton, and B. Goldstein, "Extending the Range of Rate Constants Available from BIACORE: Interpreting Mass Transport-Influenced Binding Data," Biophysical Journal, vol. 75, pp. 583-594, 1998. 